\title{
Coincidence point theorems for some multi-valued mappings in complete metric spaces endowed with a graph
}

\section{Chalongchai Klanarong and Suthep Suantai*}

\section{"Correspondence:}

suthep.s@cmu.ac.th

Department of Mathematics,

Faculty of Science, Chiang Mai

University, Chiang Mai, 50200,

Thailand

\begin{abstract}
In this paper, we introduce the concepts of weak $g$-graph-preserving for multi-valued mappings and weak $G$-contractions in a metric space endowed with a directed graph. We establish the coincidence point theorems for this type of mappings in a complete metric space endowed with a directed graph. Examples illustrating our main results are also presented. Our results extend and generalize various known results in the literature.
\end{abstract}

MSC: $47 \mathrm{H} 10 ; 54 \mathrm{H} 25$

Keywords: fixed point; coupled fixed point; multi-valued mappings; partially ordered set; graph-preserving

\section{Introduction}

Fixed point theorems for contraction mappings and their generalizations play an important role in the study of theory of equations. The Banach contraction principle [1] is a fundamental result which can be applied widely for solving the existence of solutions of various equations. Over the years, it has been widely extended and generalized in different directions by many authors (see [1-10]).

Let $(X, d)$ be a complete metric space. A mapping $T: X \rightarrow X$ is called a contraction if there exists $k \in[0,1)$ such that

$$
d(T x, T y) \leq k d(x, y) \quad \text { for all } x, y \in X .
$$

A mapping $T: X \rightarrow X$ is called a Kannan mapping if there exists a real number $\alpha \in\left[0, \frac{1}{2}\right)$ such that for every $x, y \in X$,

$$
d(T x, T y) \leq \alpha d(x, T x)+\alpha d(y, T y) .
$$

In 1968, Kannan [6] established the fixed point theorem for the Kannan mapping $T$.

One year later, Chatterjea [5] introduced the new concept of contraction mapping. Based on the Kannan mapping, he changed some variables in (1.2) and obtained new condition, that is,

$$
d(T x, T y) \leq \alpha d(x, T y)+\alpha d(y, T x) .
$$

(c) 2015 Klanarong and Suantai. This article is distributed under the terms of the Creative Commons Attribution 4.0 International License (http://creativecommons.org/licenses/by/4.0/), which permits unrestricted use, distribution, and reproduction in any medium, provided you give appropriate credit to the original author(s) and the source, provide a link to the Creative Commons license, and indicate if changes were made. 
It is easily seen that conditions (1.1) and (1.2) are independent from each other, (1.1) and (1.3) as well.

Zamfirescu [10] proved some fixed point theorems by combining (1.1), (1.2) and (1.3), which is stated as follows.

Theorem 1.1 [10] Let $(X, d)$ be a complete metric space and $T: X \rightarrow X$ be a map for which there exist the real numbers $a, b$ and $c$ satisfying $0 \leq a<1,0<b, c<\frac{1}{2}$ such that for each pair $x, y \in X$, at least one of the following is true:

(i) $d(T x, T y) \leq a d(x, y)$;

(ii) $d(T x, T y) \leq b d(x, T x)+b d(y, T y)$;

(iii) $d(T x, T y) \leq c d(x, T y)+c d(y, T x)$.

Then $T$ is a Picard operator, that is, $T$ has a unique fixed point $x_{0} \in X$ and for each $x \in X$, $T^{n} x \rightarrow x_{0}$.

In [2] Berinde introduced and studied a weak contraction mapping on a complete metric space which is weaker than the Zamfirescu operators.

Definition 1.2 [2] Let $(X, d)$ be a metric space. A map $T: X \rightarrow X$ is called weak contraction if there exist a constant $\alpha \in(0,1)$ and $L \geq 0$ such that

$$
d(T x, T y) \leq \alpha d(x, y)+L d(x, T y) \quad \text { for all } x, y \in X .
$$

The following results can be found in [2].

\section{Proposition 1.3 [2]}

(1) Any Kannan mapping is a weak contraction.

(2) Any mapping $T$ satisfying the contractive condition (1.3) is a weak contraction.

(3) Any Zamfirescu mapping, i.e., any mapping satisfying the assumptions in Theorem 1.1, is a weak contraction.

A partial ordering is a binary relation $\preceq$ over the set $X$ which satisfies the following conditions:

(1) $x \preceq x$ (reflexivity);

(2) $x \preceq y$ and $y \preceq x$, then $x=y$ (antisymmetry);

(3) $x \preceq y$ and $y \preceq z$, then $x \preceq z$ (transitivity)

for all $x, y, z \in X$. A set with a partial ordering $\preceq$ is called a partially ordered set. We write $x \prec y$ if $x \preceq y$ and $x \neq y$.

Fixed point theorems for monotone single-valued mappings have been investigated and studied in partially ordered metric spaces by many mathematicians (see [7-9, 11, 12]). Nieto and Rodriguez-Lopez $[7,8]$ were the first who studied some fixed point theorems for monotone nondecreasing mappings in partially ordered metric spaces and applied the obtained results to study an existence problem of ordinary differential equations.

The study of fixed point for multi-valued contraction mappings using the PompeiuHausdorff metric was first performed by Nadler [13].

Let $(X, d)$ be a metric space. For $x \in X$ and $A \subseteq X$, we denote $D(x, A)=\inf \{d(x, y): y \in A\}$. The class of all nonempty bounded and closed subsets of $X$ is denoted by $C B(X)$. Let $H$ be 
a Pompeiu-Hausdorff metric induced by the metric $d$ on $X$, that is,

$$
H(A, B)=\max \left\{\sup _{x \in A} D(x, B), \sup _{y \in B} D(y, A)\right\}
$$

for every $A, B \in C B(X)$.

We let $\operatorname{Comp}(X)$ be the set of all nonempty compact subsets of $X$. It is clear that $\operatorname{Comp}(X)$ is included in $C B(X)$.

Let $T: X \rightarrow 2^{X}$ (collection of all nonempty subsets of $X$ ) and $g: X \rightarrow X$. An element $x \in X$ is called

(i) fixed point of $T$ if $x \in T(x)$,

(ii) coincidence point of a hybrid pair $\{T, g\}$ if $g(x) \in T(x)$.

In 1969, Nadler [13] introduced the concept of Banach contraction principle for a multivalued mapping and proved the existence of fixed point for multi-valued version of the Banach contraction principle. The following theorem is the first well-known theorem of multi-valued contractions studied by Nadler.

Theorem 1.4 [13] Let $(X, d)$ be a complete metric space and $T: X \rightarrow C B(X)$ be a multivalued mapping. If there exists $k \in[0,1)$ such that

$$
H(T x, T y) \leq k d(x, y) \quad \text { for all } x, y \in X
$$

then $T$ has a fixed point in $X$.

In 2007, Berinde and Berinde [14] provided the new type of contraction which is a generalization of the contraction principle considered by Nadler.

Definition 1.5 [14] Let $(X, d)$ be a metric space and $T: X \rightarrow C B(X)$ be a multi-valued mapping. $T$ is said to be a multi-valued weak contraction or a multi-valued $(\theta, L)$-weak contraction if there exist two constants $\theta \in(0,1)$ and $L \geq 0$ such that

$$
H(T x, T y) \leq \theta d(x, y)+L d(y, T x) \quad \text { for all } x, y \in X .
$$

We now recall some notions concerning a directed graph. Let $(X, d)$ be a metric space and $\Delta$ denote the diagonal of $X \times X$. Let $G$ be a directed graph such that the set $V(G)$ of its vertices coincides with $X$ and the set $E(G)$ of its edges is a subset of $X \times X$. We assume that the graph $G$ has no parallel edges and, thus, one can identify $G$ with the pair $(V(G), E(G))$. We denote by $G^{-1}$ the conversion of a graph $G$, i.e.,

$$
G^{-1}=\{(x, y) \in X \times X:(y, x) \in E(G)\} .
$$

The next definition, G-contraction, was introduced by Jachymski [15] in 2008.

Definition 1.6 [15] Let $(X, d)$ be a metric space and $G=(V(G), E(G))$ be a directed graph such that $V(G)=X$ and $E(G)$ contains all loops, i.e., $\Delta \subseteq E(G)$. We say that a mapping $T: X \rightarrow X$ is a $G$-contraction if $T$ preserves edges of $G$, i.e., for every $x, y \in X$,

$$
(x, y) \in E(G) \quad \Rightarrow \quad(T x, T y) \in E(G)
$$


and there exists $\alpha \in(0,1)$ such that $x, y \in X$,

$$
(x, y) \in E(G) \quad \Rightarrow \quad d(T x, T y) \leq \alpha d(x, y) .
$$

The mapping $T: X \rightarrow X$ satisfying condition (1.5) is called a graph-preserving mapping. Under some additional properties of a metric space $X$ endowed with a directed graph, Jachymski showed that a $G$-contraction $T: X \rightarrow X$ has a fixed point if and only if there exists $x \in X$ such that $(x, T(x)) \in E(G)$.

Subsequently, Beg and Butt [16] tried to introduce the concept of G-contraction for multi-valued mappings, but their extension was not carried correctly (see $[17,18]$ ).

In 2011, Nicolae et al. [19] extended the notion of multivalued contraction on a metric space with a graph.

Recently, Dinevari and Frigon [20] introduced a new concept of G-contraction multivalued mappings.

Definition 1.7 [20] Let $T: X \rightarrow 2^{X}$ be a map with nonempty values. We say that $T$ is a G-contraction (in the sense of Dinevari and Frigon) if there exists $\alpha \in(0,1)$ such that for all $(x, y) \in E(G)$ and $u \in T x$, there exists $v \in T y$ such that $(u, v) \in E(G)$ and $d(u, v) \leq \alpha d(x, y)$.

They showed that under some properties, weaker than Property (A), a multi-valued Gcontraction with the closed value has a fixed point. Recently, Tiammee and Suantai [21] introduced the concept of graph-preserving for multi-valued mappings and proved their fixed point theorem in a complete metric space endowed with a graph.

Definition 1.8 [21] Let $X$ be a nonempty set, $G=(V(G), E(G))$ be a directed graph such that $V(G)=X$, and $T: X \rightarrow C B(X)$. Then $T$ is said to be graph-preserving if $(x, y) \in E(G) \Rightarrow$ $(u, v) \in E(G)$ for all $u \in T x$ and $v \in T y$.

Definition 1.9 [21] Let $X$ be a nonempty set, $G=(V(G), E(G))$ be a directed graph such that $V(G)=X, g: X \rightarrow X$, and $T: X \rightarrow C B(X)$. Then $T$ is said to be $g$-graph-preserving if for any $x, y \in X$ such that

$$
(g(x), g(y)) \in E(G) \quad \Rightarrow \quad(u, v) \in E(G)
$$

for all $u \in T x$ and $v \in T y$.

Recently, Phon-on et al. [22] introduced a new type of weak G-contraction which is weaker than that of Tiammee and Suantai [21], and they proved some fixed point theorems for this type of mappings with compact values which is a generalization of several known results in a complete metric space endowed with a graph.

Definition 1.10 [22] Let $X$ be a nonempty set and $G=(V(G), E(G))$ be a directed graph such that $V(G)=X$, and $T: X \rightarrow \operatorname{Comp}(X)$. Then $T$ is said to be weak graph-preserving if it satisfies the following: for each $x, y \in X$, if $(x, y) \in E(G)$, then for each $u \in T x$ there is $v \in P_{T y}(u)$ such that $(u, v) \in E(G)$, where $P_{T y}(u)=\{a \in T y \mid d(u, a)=D(u, T y)\}$.

Motivated and inspired by all of those works mentioned above, we aim to introduce a new type of multi-valued contractions which is more general than that of Berinde [2]. 
These classes of mappings are defined for multi-valued mappings in complete metric spaces endowed with directed graphs. Fixed point theorems for this type of mappings are established. We also apply our main results for proving the existence theorem of a fixed point for a multi-valued mapping defined on a partially ordered metric space. Moreover, we also apply the obtained results to prove the existence theorem of a coupled fixed point. Some examples are given to illustrate our results.

\section{Preliminaries}

Let $(X, d)$ be a metric space and let $C B(X)$ be the class of all nonempty bounded and closed subsets of $X$. For $x \in X$ and $A \subseteq X$, we denote

$$
D(x, A)=\inf \{d(x, y) \mid y \in A\} .
$$

Let $H$ be a Pompeiu-Hausdorff metric induced by the metric $d$ on $X$, that is,

$$
H(A, B)=\max \left\{\sup _{x \in A} D(x, B), \sup _{y \in B} D(y, A)\right\}
$$

for every $A, B \in C B(X)$. An element $y_{0} \in A$ is said to be a best approximation to $x$ if

$$
d\left(x, y_{0}\right)=D(x, A)
$$

A nonempty subset $A$ of $X$ is a proximinal set if for each $x \in X$, there exists a point $y \in A$ such that $d(x, y)=D(x, A)$. Let $P B(X)$ be the family of all bounded proximinal subsets of $X$. The set of all best approximations from $x$ to $A$ is denoted by

$$
P_{A}(x)=\{y \in A \mid d(x, y)=D(x, A)\} .
$$

This defines a mapping $P_{A}: X \rightarrow 2^{A}$ and is called the metric projection into $A$. It is clear that $P B(X)$ is included in $C B(X)$.

The following lemma is useful for our main results.

Lemma 2.1 [13] Let $A, B \in C B(X)$ and $a \in A$. Then, for $\varepsilon>0$, there exists an element $b \in B$ such that $d(a, b) \leq H(A, B)+\varepsilon$.

Definition 2.2 Let $X$ be a nonempty set, $G=(V(G), E(G))$ be a directed graph such that $V(G)=X$ and let $T: X \rightarrow P B(X)$ and $g: X \rightarrow X$. Then $T$ is said to be weak $g$-graphpreserving if for any $x, y \in X$ such that $(g(x), g(y)) \in E(G)$, then for each $u \in T x$ there exists $v \in P_{T y}(u)$ such that $(u, v) \in E(G)$.

Example 2.3 Let $X=\{0,1\} \cup\left\{\frac{1}{2^{n}} \mid n \in \mathbb{N}\right\}$ and let $d$ be a usual metric on $X$. Let $G=$ $(V(G), E(G))$ be a directed graph defined by $V(G)=X$ and

$$
\begin{aligned}
E(G)= & \left\{(1,0),\left(1, \frac{1}{2}\right),(1,1)\right\} \cup\left\{\left(\frac{1}{2^{n}}, \frac{1}{2^{n+1}}\right) \mid n \in \mathbb{N}\right\} \\
& \cup\left\{\left(\frac{1}{2^{n}}, 0\right),\left(\frac{1}{2^{n}}, \frac{1}{2^{n}}\right) \mid n \in \mathbb{N}\right\} .
\end{aligned}
$$


Let $T: X \rightarrow P B(X)$ and $g: X \rightarrow X$ be defined by

$$
T x= \begin{cases}\{0,1\} & \text { if } x=0 \\ \left\{\frac{1}{2^{n+1}}, 1\right\} & \text { if } x=\frac{1}{2^{n}}, n \in \mathbb{N} \\ \left\{\frac{1}{2}, 1\right\} & \text { if } x=1,\end{cases}
$$

and $g(x)=x^{2}$.

Now we will show that $T$ is weak $g$-graph-preserving. Let $(g(x), g(y)) \in E(G)$.

If $(g(x), g(y))=(1,0)$, then $(x, y)=(1,0), T x=\left\{\frac{1}{2}, 1\right\}$, and $T y=\{0,1\}$, we have $P_{T y}\left(\frac{1}{2}\right)=\{0,1\}$, $P_{T y}(1)=\{1\}$ and $\left(\frac{1}{2}, 0\right),(1,1) \in E(G)$.

If $(g(x), g(y))=\left(\frac{1}{2^{2 n}}, \frac{1}{2^{2 n}}\right)$, then $(x, y)=\left(\frac{1}{2^{n}}, \frac{1}{2^{n}}\right)$ and $T x=\left\{\frac{1}{2^{n+1}}, 1\right\}=T y$, we have $P_{T y}(1)=\{1\}$, $P_{T y}\left(\frac{1}{2^{n+1}}\right)=\left\{\frac{1}{2^{n+1}}\right\}$ and $(1,1),\left(\frac{1}{2^{n+1}}, \frac{1}{2^{n+1}}\right) \in E(G)$.

If $(g(x), g(y))=\left(\frac{1}{2^{2 n}}, 0\right)$, then $(x, y)=\left(\frac{1}{2^{n}}, 0\right), T x=\left\{\frac{1}{2^{n+1}}, 1\right\}$, and $T y=\{0,1\}$, we have $P_{T y}\left(\frac{1}{2^{n+1}}\right)=\{0\}, P_{T y}(1)=\{1\}$ and $\left(\frac{1}{2^{n+1}}, 0\right),(1,1) \in E(G)$.

If $(g(x), g(y))=(1,1)$, then $(x, y)=(1,1)$ and $T x=\left\{\frac{1}{2}, 1\right\}=T y$, we have $P_{T y}\left(\frac{1}{2}\right)=\left\{\frac{1}{2}\right\}, P_{T y}(1)=$ $\{1\}$ and $\left(\frac{1}{2}, \frac{1}{2}\right),(1,1) \in E(G)$.

Hence $T$ is weak $g$-graph-preserving.

Definition 2.4 Let $X$ be a nonempty set, $G=(V(G), E(G))$ be a directed graph such that $V(G)=X$ and let $T: X \rightarrow X$ and $g: X \rightarrow X$ be self-mappings on $X$. Then $T$ is said to be G-edge-preserving with respect to $g$ if for any $x, y \in X$,

$$
(g(x), g(y)) \in E(G) \quad \text { implies } \quad(T x, T y) \in E(G) .
$$

\section{Main results}

We start with introducing a new type of weak $G$-contraction with respect to $g$.

Definition 3.1 Let $(X, d)$ be a metric space, $G=(V(G), E(G))$ be a directed graph such that $V(G)=X, g: X \rightarrow X$ and $T: X \rightarrow P B(X)$. $T$ is said to be a $(k, L, r)$ weak $G$-contraction with respect to $g$ if there exist $L \geq 0$ and $k, r \in[0,1)$ with $0<k+r<1$ such that

$$
H(T x, T y) \leq k d(g(x), g(y))+L D(g(y), T x)+r D(g(x), T y)
$$

for all $x, y \in X$ with $(g(x), g(y)) \in E(G)$.

Example 3.2 Let $X=\{0,1\} \cup\left\{\frac{1}{2^{n}} \mid n \in \mathbb{N}\right\}$ and let $d$ be a usual metric on $X$. Let $G=$ $(V(G), E(G))$ be a directed graph defined by $V(G)=X$ and

$$
\begin{aligned}
E(G)= & \left\{(0,0),(1,0),\left(1, \frac{1}{2}\right)\right\} \cup\left\{\left(\frac{1}{2^{n}}, \frac{1}{2^{n+1}}\right) \mid n \in \mathbb{N}\right\} \\
& \cup\left\{\left(\frac{1}{2^{n}}, 0\right),\left(\frac{1}{2^{n}}, \frac{1}{2^{n}}\right) \mid n \in \mathbb{N}\right\} .
\end{aligned}
$$

Let $T: X \rightarrow P B(X)$ and $g: X \rightarrow X$ be defined by

$$
T x= \begin{cases}\{0\} & \text { if } x=1 \\ \left\{\frac{1}{2^{n+1}}, 0\right\} & \text { if } x=\frac{1}{2^{n}}, n \in \mathbb{N} \\ \left\{\frac{1}{2}\right\} & \text { if } x=0,\end{cases}
$$


and

$$
g(x)= \begin{cases}1 & \text { if } x=0,1, \frac{1}{2} \\ \frac{1}{2^{n-1}} & \text { if } x=\frac{1}{2^{n}}, n \in \mathbb{N} \backslash\{1\} .\end{cases}
$$

Now we prove that $T$ is a $(k, L, r)$ weak $G$-contraction with respect to $g$, where $k=\frac{1}{2}$, $\frac{2}{7} \leq r<\frac{1}{2}$, and $L \geq 0$. Let $x, y \in X$ such that $(x, y) \in E(G)$.

If $(g(x), g(y))=\left(\frac{1}{2^{n-1}}, \frac{1}{2^{n-1}}\right), n \neq 1$. Then $H(T x, T y)=0$, so

$$
H(T x, T y) \leq k d(g(x), g(y))+L D(g(y), T x)+r D(g(x), T y) .
$$

If $(g(x), g(y))=\left(1, \frac{1}{2}\right)$, then $(x, y)=\left(1, \frac{1}{4}\right),\left(0, \frac{1}{4}\right),\left(\frac{1}{2}, \frac{1}{4}\right)$.

Case $(x, y)=\left(1, \frac{1}{4}\right)$, we have $T x=\{0\}$ and $T y=\left\{\frac{1}{8}, 0\right\}$. Thus

$$
H(T x, T y)=\frac{1}{8} \leq \frac{1}{2}(g(x), g(y))+L D(g(y), T x)+r D(g(x), T y) .
$$

Case $(x, y)=\left(0, \frac{1}{4}\right)$, we have $T x=\left\{\frac{1}{2}\right\}$ and $T y=\left\{\frac{1}{8}, 0\right\}$. Thus

$$
H(T x, T y)=\frac{1}{2} \leq \frac{1}{2}(g(x), g(y))+L D(g(y), T x)+r D(g(x), T y) .
$$

Case $(x, y)=\left(\frac{1}{2}, \frac{1}{4}\right)$, we have $T x=\left\{\frac{1}{4}, 0\right\}$ and $T y=\left\{\frac{1}{8}, 0\right\}$. Thus

$$
H(T x, T y)=\frac{1}{8} \leq \frac{1}{2}(g(x), g(y))+L D(g(y), T x)+r D(g(x), T y) .
$$

If $(g(x), g(y))=\left(\frac{1}{2^{n-1}}, \frac{1}{2^{n}}\right), n \neq 1$, then $(x, y)=\left(\frac{1}{2^{n}}, \frac{1}{2^{n+1}}\right)$. We have $T x=\left\{\frac{1}{2^{n+1}}, 0\right\}$ and $T y=$ $\left\{\frac{1}{2^{n+2}}, 0\right\}$. Thus

$$
H(T x, T y)=\frac{1}{2^{n+2}}=\frac{1}{2}\left(\frac{1}{2^{n+1}}\right) \leq \frac{1}{2}(g(x), g(y))+L D(g(y), T x)+r D(g(x), T y) .
$$

Hence $T$ is a $(k, L, r)$ weak $G$-contraction with respect to $g$.

The following property is useful for our main results.

Property (A) [15] For any sequence $\left(x_{n}\right)_{n \in \mathbb{N}}$ in $X$, if $x_{n} \rightarrow x$ and $\left(x_{n}, x_{n+1}\right) \in E(G)$ for $n \in \mathbb{N}$, then there is a subsequence $\left(x_{k_{n}}\right)_{n \in \mathbb{N}}$ with $\left(x_{k_{n}}, x\right) \in E(G)$ for $n \in \mathbb{N}$.

We first prove our main result.

Theorem 3.3 Let $(X, d)$ be a complete metric space, $G=(V(G), E(G))$ be a directed graph such that $V(G)=X$, and let $g: X \rightarrow X$ be a surjective mapping. If $T: X \rightarrow P B(X)$ is a multi-valued mapping satisfying the following properties:

(i) $T$ is a weak g-graph-preserving mapping;

(ii) there exists $x_{0} \in X$ such that $\left(g\left(x_{0}\right), y\right) \in E(G)$ for some $y \in T x_{0}$;

(iii) $X$ has Property $(A)$;

(iv) $T$ is a $(k, L, r)$ weak $G$-contraction,

then there exists $u \in X$ such that $g(u) \in T u$. 
Proof Let $x_{0} \in X$ be such that $\left(g\left(x_{0}\right), y\right) \in E(G)$ for some $y \in T x_{0}$. Since $g$ is surjective, there exists $x_{1} \in X$ such that $y=g\left(x_{1}\right) \in T x_{0}$. By (ii), we have $\left(g\left(x_{0}\right), g\left(x_{1}\right)\right) \in E(G)$. Since $T$ is weak $g$-graph-preserving and $g$ is surjective, there exists $x_{2} \in X$ such that $g\left(x_{2}\right) \in P_{T x_{1}}\left(g\left(x_{1}\right)\right)$ and $\left(g\left(x_{1}\right), g\left(x_{2}\right)\right) \in E(G)$. By Lemma 2.1, there exists $y_{1} \in T x_{1}$ such that

$$
d\left(g\left(x_{1}\right), y_{1}\right) \leq H\left(T x_{0}, T x_{1}\right)+\frac{1}{2} .
$$

It follows that

$$
\begin{aligned}
d\left(g\left(x_{1}\right), g\left(x_{2}\right)\right) & =D\left(g\left(x_{1}\right), T x_{1}\right) \\
& \leq d\left(g\left(x_{1}\right), y_{1}\right) \\
& \leq H\left(T x_{0}, T x_{1}\right)+\frac{1}{2} .
\end{aligned}
$$

Since $\left(g\left(x_{0}\right), g\left(x_{1}\right)\right) \in E(G)$ and $T$ is a $(k, L, r)$ weak $G$-contraction, we have

$$
\begin{aligned}
H\left(T x_{0}, T x_{1}\right) & \leq k d\left(x_{0}\right), g\left(x_{1}\right)+L D\left(g\left(x_{1}\right), T x_{0}\right)+r D\left(g\left(x_{0}\right), T x_{1}\right) \\
& =r D\left(g\left(x_{0}\right), T x_{1}\right)+k d\left(g\left(x_{0}\right), g\left(x_{1}\right)\right) \\
& \leq r d\left(g\left(x_{0}\right), g\left(x_{2}\right)\right)+k d\left(g\left(x_{0}\right), g\left(x_{1}\right)\right) \\
& \leq r\left[d\left(g\left(x_{0}\right), g\left(x_{1}\right)\right)+d\left(g\left(x_{1}\right), g\left(x_{2}\right)\right)\right]+k d\left(g\left(x_{0}\right), g\left(x_{1}\right)\right) \\
& =(k+r) d\left(g\left(x_{0}\right), g\left(x_{1}\right)\right)+r d\left(g\left(x_{1}\right), g\left(x_{2}\right)\right) \\
& =(k+r) d\left(g\left(x_{0}\right), g\left(x_{1}\right)\right)+r D\left(g\left(x_{1}\right), T x_{1}\right) \\
& \leq(k+r) d\left(g\left(x_{0}\right), g\left(x_{1}\right)\right)+r H\left(T x_{0}, T x_{1}\right),
\end{aligned}
$$

which implies

$$
H\left(T x_{0}, T x_{1}\right) \leq \frac{k+r}{1-r} d\left(g\left(x_{0}\right), g\left(x_{1}\right)\right) .
$$

From this together with (3.1), we obtain

$$
d\left(g\left(x_{1}\right), g\left(x_{2}\right)\right) \leq \frac{k+r}{1-r} d\left(g\left(x_{0}\right), g\left(x_{1}\right)\right)+\frac{1}{2} .
$$

Since $T$ is weak $g$-graph-preserving and $g$ is surjective, there exists $x_{3} \in X$ such that $g\left(x_{3}\right) \in$ $P_{T x_{2}}\left(g\left(x_{2}\right)\right)$ and $\left(g\left(x_{2}\right), g\left(x_{3}\right)\right) \in E(G)$. By Lemma 2.1, there exists $y_{2} \in T x_{2}$ such that

$$
d\left(g\left(x_{2}\right), y_{2}\right) \leq H\left(T x_{1}, T x_{2}\right)+\frac{1}{2^{2}} .
$$

It follows that

$$
\begin{aligned}
d\left(g\left(x_{2}\right), g\left(x_{3}\right)\right) & =D\left(g\left(x_{2}\right), T x_{2}\right) \\
& \leq d\left(g\left(x_{2}\right), y_{2}\right) \\
& \leq H\left(T x_{1}, T x_{2}\right)+\frac{1}{2^{2}} .
\end{aligned}
$$


Since $T$ is a $(k, L, r)$ weak $G$-contraction, by (3.3), we get

$$
\begin{aligned}
H\left(T x_{1}, T x_{2}\right) & \leq k d\left(g\left(x_{1}\right), g\left(x_{2}\right)\right)+L D\left(g\left(x_{2}\right), T x_{1}\right)+r D\left(g\left(x_{1}\right), T x_{2}\right) \\
& =r D\left(g\left(x_{1}\right), T x_{2}\right)+k d\left(g\left(x_{1}\right), g\left(x_{2}\right)\right) \\
& \leq r d\left(g\left(x_{1}\right), g\left(x_{3}\right)\right)+k d\left(g\left(x_{1}\right), g\left(x_{2}\right)\right) \\
& \leq r\left[d\left(g\left(x_{1}\right), g\left(x_{2}\right)\right)+d\left(g\left(x_{2}\right), g\left(x_{3}\right)\right)\right]+k d\left(g\left(x_{1}\right), g\left(x_{2}\right)\right) \\
& =(r+k) d\left(g\left(x_{1}\right), g\left(x_{2}\right)\right)+r d\left(g\left(x_{2}\right), g\left(x_{3}\right)\right) \\
& \leq(k+r) d\left(g\left(x_{1}\right), g\left(x_{2}\right)\right)+r D\left(g\left(x_{2}\right), T x_{2}\right) \\
& \leq(k+r) d\left(g\left(x_{1}\right), g\left(x_{2}\right)\right)+r H\left(T x_{1}, T x_{2}\right) .
\end{aligned}
$$

This implies

$$
H\left(T x_{1}, T x_{2}\right) \leq \frac{k+r}{1-r} d\left(g\left(x_{1}\right), g\left(x_{2}\right)\right) .
$$

From this together with (3.3), we obtain

$$
\begin{aligned}
d\left(g\left(x_{2}\right), g\left(x_{3}\right)\right) & \leq\left(\frac{k+r}{1-r}\right) d\left(g\left(x_{1}\right), g\left(x_{2}\right)\right)+\frac{1}{2^{2}} \\
& =\left(\frac{k+r}{1-r}\right) D\left(g\left(x_{1}\right), T\left(x_{1}\right)\right)+\frac{1}{2^{2}} \\
& \leq\left(\frac{k+r}{1-r}\right) H\left(T\left(x_{0}\right), T\left(x_{1}\right)\right)+\frac{1}{2^{2}} \\
& \leq\left(\frac{k+r}{1-r}\right)^{2} d\left(g\left(x_{0}\right), g\left(x_{1}\right)\right)+\frac{1}{2^{2}} .
\end{aligned}
$$

By induction, we obtain a sequence $\left\{g\left(x_{n}\right)\right\}$ in $X$ such that for each $n \in \mathbb{N}, g\left(x_{n+1}\right) \in T x_{n}$, $\left(g\left(x_{n}\right), g\left(x_{n+1}\right)\right) \in E(G)$ and

$$
d\left(g\left(x_{n}\right), g\left(x_{n+1}\right)\right) \leq\left(\frac{k+r}{1-r}\right)^{n} d\left(g\left(x_{0}\right), g\left(x_{1}\right)\right)+\frac{1}{2^{n}} .
$$

Put $q=\frac{k+r}{1-r}$. For any $n, m \in \mathbb{N}$ with $m>n$, by using (3.4), we have

$$
\begin{aligned}
d\left(g\left(x_{n}\right), g\left(x_{m}\right)\right) \leq & d\left(g\left(x_{n}\right), g\left(x_{n+1}\right)\right)+d\left(g\left(x_{n+1}\right), g\left(x_{n+2}\right)\right)+\cdots+d\left(g\left(x_{m-1}\right), g\left(x_{m}\right)\right) \\
\leq & q^{n} d\left(g\left(x_{0}\right), g\left(x_{1}\right)\right)+\frac{1}{2^{n}}+q^{n+1} d\left(g\left(x_{0}\right), g\left(x_{1}\right)\right)+\frac{1}{2^{n+1}}+\cdots \\
& +q^{m-1} d\left(g\left(x_{0}\right), g\left(x_{1}\right)\right)+\frac{1}{2^{m-1}} \\
\leq & d\left(g\left(x_{0}\right), g\left(x_{1}\right)\right) \sum_{i=n}^{m-1} q^{i}+\sum_{i=n}^{m-1} \frac{1}{2^{i}} \\
\leq & \frac{q^{n}}{1-q} d\left(g\left(x_{0}\right), g\left(x_{1}\right)\right)+\frac{2}{2^{n}}
\end{aligned}
$$


which, in view of $0<q<1$, implies that $\left\{g\left(x_{n}\right)\right\}$ is a Cauchy sequence. Since $X$ is complete, there exists $u \in X$ such that $\lim _{n \rightarrow \infty} g\left(x_{n}\right)=g(u)$. By assumption (iii), there is a subsequence $\left\{g\left(x_{n_{k}}\right)\right\}$ of $\left\{g\left(x_{n}\right)\right\}$ such that $\left(g\left(x_{n_{k}}, g(u)\right)\right) \in E(G)$ for all $k \in \mathbb{N}$. Since $T$ is a weak- $G$ contraction, we have

$$
\begin{aligned}
D(g(u), T u) & \leq d\left(g(u), g\left(x_{n_{k}+1}\right)\right)+D\left(g\left(x_{n_{k}+1}\right), T u\right) \\
& \leq d\left(g(u), g\left(x_{n_{k}+1}\right)\right)+H\left(T x_{n_{k}}, T u\right) \\
& \leq d\left(g(u), g\left(x_{n_{k}+1}\right)\right)+k d\left(g\left(x_{n_{k}}\right), g(u)\right)+L D\left(g(u), T x_{n_{k}}\right)+r D\left(g\left(x_{n_{k}}\right), T u\right) \\
& \leq d\left(g(u), g\left(x_{n_{k}+1}\right)\right)+k d\left(g\left(x_{n_{k}}\right), g(u)\right)+L d\left(g(u), g\left(x_{n_{k}+1}\right)\right)+r D\left(g\left(x_{n_{k}}\right), T u\right) .
\end{aligned}
$$

Let $y \in T u$. From the above inequality, we get

$$
\begin{aligned}
D(g(u), T u) \leq & d\left(g(u), g\left(x_{n_{k}+1}\right)\right)+k d\left(g\left(x_{n_{k}}\right), g(u)\right) \\
& +L d\left(g(u), g\left(x_{n_{k}+1}\right)\right)+r d\left(g\left(x_{n_{k}}\right), y\right) .
\end{aligned}
$$

Letting $n \rightarrow \infty$ in the above inequality, we obtain

$$
D(g(u), T u) \leq r d(g(u), y)
$$

Since y is arbitrary, it follows that $D(g(u), T u) \leq r D(g(u), T u)$, which implies $D(g(u), T u)=0$. As $T u$ is closed, $g(u) \in T u$. This completes the proof.

The following result is obtained directly from Theorem 3.3 in case that $T$ is a singlevalued mapping.

Theorem 3.4 Let $(X, d)$ be a complete metric space and $G=(V(G), E(G))$ be a directed graph such that $V(G)=X$, and let $g: X \rightarrow X$ be a surjective mapping. Let $T: X \rightarrow X$ be a single-valued mapping satisfying the following properties:

(i) $T$ is a G-edge-preserving mapping with respect to $g$;

(ii) there exists $x_{0} \in X$ such that $\left(g\left(x_{0}\right), T x_{0}\right) \in E(G)$;

(iii) $X$ has Property $(A)$;

(iv) there exist $L \geq 0$ and $k, r \in[0,1)$ with $0<k+r<1$ such that

$$
d(T x, T y) \leq k d(g(x), g(y))+L d(g(y), T x)+r d(g(x), T y)
$$

for all $x, y \in X$ with $(g(x), g(y)) \in E(G)$,

then there exists $u \in X$ such that $g(u) \in T u$.

Example 3.5 Let $X=\{0,1\} \cup\left\{\frac{1}{2^{n}} \mid n \in \mathbb{N}\right\}$ and let $d$ be a usual metric on $X$. Let $G=$ $(V(G), E(G))$ be a directed graph defined by $V(G)=X$ and

$$
\begin{aligned}
E(G)= & \{(0,0),(1,0)\} \cup\left\{\left(\frac{1}{2^{n}}, \frac{1}{2^{n+1}}\right) \mid n \in \mathbb{N}\right\} \\
& \cup\left\{\left(\frac{1}{2^{n}}, 0\right),\left(\frac{1}{2^{n}}, \frac{1}{2^{n}}\right),\left(1, \frac{1}{2^{n}}\right) \mid n \in \mathbb{N}\right\} .
\end{aligned}
$$


Let $T: X \rightarrow P B(X)$ and $g: X \rightarrow X$ be defined by

$$
T x= \begin{cases}\{1\} & \text { if } x=0 \\ \left\{\frac{1}{2^{n+1}}, 0\right\} & \text { if } x=\frac{1}{2^{n}}, n \in \mathbb{N} \\ \{0\} & \text { if } x=1,\end{cases}
$$

and

$$
g(x)= \begin{cases}1 & \text { if } x=0 \\ \frac{1}{2^{n}} & \text { if } x=\frac{1}{2^{n}}, n \in \mathbb{N} \\ 0 & \text { if } x=1\end{cases}
$$

Now we will show that $T$ is weak $g$-graph-preserving. Let $(g(x), g(y)) \in E(G)$.

If $(g(x), g(y))=(0,0)$, then $(x, y)=(1,1)$ and so $T x=\{0\}=T y$. We have $P_{T y}(0)=\{0\}$ and $(0,0) \in E(G)$.

If $(g(x), g(y))=(1,0)$, then $(x, y)=(0,1)$ and so $T x=\{1\}, T y=\{0\}$. We have $P_{T y}(1)=\{0\}$ and $(1,0) \in E(G)$.

If $(g(x), g(y))=\left(1, \frac{1}{2^{n}}\right)$, then $(x, y)=\left(0, \frac{1}{2^{n}}\right)$ and so $T x=\{1\}, T y=\left\{\frac{1}{2^{n+1}}, 0\right\}$. We have $P_{T y}(1)=$ $\left\{\frac{1}{2^{n+1}}\right\}$ and $\left(1, \frac{1}{2^{n+1}}\right) \in E(G)$.

If $(g(x), g(y))=\left(\frac{1}{2^{n}}, \frac{1}{2^{n}}\right)$, then $(x, y)=\left(\frac{1}{2^{n}}, \frac{1}{2^{n}}\right)$ and so $T x=\left\{\frac{1}{2^{n+1}}, 0\right\}=T y$. We have $P_{T y}(0)=$ $\{0\}, P_{T y}\left(\frac{1}{2^{n+1}}\right)=\left\{\frac{1}{2^{n+1}}\right\}$ and $(0,0),\left(\frac{1}{2^{n+1}}, \frac{1}{2^{n+1}}\right) \in E(G)$.

If $(g(x), g(y))=\left(\frac{1}{2^{n}}, 0\right)$, then $(x, y)=\left(\frac{1}{2^{n}}, 1\right)$ and so $T x=\left\{\frac{1}{2^{n+1}}, 0\right\}, T y=\{0\}$. We have $P_{T y}(0)=$ $\{0\}=P_{T y}\left(\frac{1}{2^{n+1}}\right)$ and $(0,0),\left(\frac{1}{2^{n+1}}, 0\right) \in E(G)$.

If $(g(x), g(y))=\left(\frac{1}{2^{n}}, \frac{1}{2^{n+1}}\right)$, then $(x, y)=\left(\frac{1}{2^{n}}, \frac{1}{2^{n+1}}\right)$ and so $T x=\left\{\frac{1}{2^{n+1}}, 0\right\}, T y=\left\{\frac{1}{2^{n+2}}, 0\right\}$. We have $P_{T y}(0)=\{0\}, P_{T y}\left(\frac{1}{2^{n+1}}\right)=\left\{\frac{1}{2^{n+1}}\right\}$ and $(0,0),\left(\frac{1}{2^{n+1}}, \frac{1}{2^{n+2}}\right) \in E(G)$.

Hence $T$ is weak $g$-graph-preserving.

Next we prove that $T$ is a $(k, L, r)$ weak $G$-contraction with respect to $g$, where $k=\frac{1}{2}$, $r=\frac{1}{4}$, and $L \geq 1$. Let $x, y \in X$ such that $(g(x), g(y)) \in E(G)$.

If $(g(x), g(y))=(0,0)$ and $(g(x), g(y))=\left(\frac{1}{2^{n}}, \frac{1}{2^{n}}\right)$, then $H(T x, T y)=0$, so

$$
H(T x, T y) \leq k d(g(x), g(y))+L D(g(y), T x)+r D(g(x), T y) .
$$

If $(g(x), g(y))=(1,0)$, then $(x, y)=(0,1)$ and so $T x=\{1\}, T y=\{0\}$. Thus

$$
H(T x, T y)=1 \leq \frac{1}{2} d(g(x), g(y))+L D(g(y), T x)+r D(g(x), T y) .
$$

If $(g(x), g(y))=\left(1, \frac{1}{2^{n}}\right)$, then $(x, y)=\left(0, \frac{1}{2^{n}}\right)$ and so $T x=\{1\}, T y=\left\{\frac{1}{2^{n+1}}, 0\right\}$. Thus

$$
H(T x, T y)=1-\frac{1}{2^{n+1}} \leq \frac{1}{2} d(g(x), g(y))+L D(g(y), T x)+r D(g(x), T y) .
$$

If $(g(x), g(y))=\left(\frac{1}{2^{n}}, 0\right)$, then $(x, y)=\left(\frac{1}{2^{n}}, 1\right)$ and so $T x=\left\{\frac{1}{2^{n+1}}, 0\right\}, T y=\{0\}$. Thus

$$
H(T x, T y)=\frac{1}{2^{n+1}}=\frac{1}{2}\left(\frac{1}{2^{n}}\right) \leq \frac{1}{2} d(g(x), g(y))+L D(g(y), T x)+r D(g(x), T y) .
$$


If $(g(x), g(y))=\left(\frac{1}{2^{n}}, \frac{1}{2^{n+1}}\right)$, then $(x, y)=\left(\frac{1}{2^{n}}, \frac{1}{2^{n+1}}\right)$ and so $T x=\left\{\frac{1}{2^{n+1}}, 0\right\}, T y=\left\{\frac{1}{2^{n+2}}, 0\right\}$. Thus

$$
H(T x, T y)=\frac{1}{2^{n+2}} \leq \frac{1}{2} d(g(x), g(y))+L D(g(y), T x)+r D(g(x), T y) .
$$

Hence $T$ is a $(k, L, r)$ weak $G$-contraction with respect to $g$. So conditions (i) and (iv) of Theorem 3.3 are satisfied. Also, it is easy to check that condition (iii) of Theorem 3.3 is satisfied. Then all the hypotheses in Theorem 3.3 are satisfied, and we see that 0 and 1 are coincidence points of $T$ and $g$.

We next apply Theorem 3.3 to obtain fixed point theorems for some contraction mappings in partially ordered metric spaces.

Definition 3.6 Let $(\mathrm{X}, \mathrm{d})$ be a metric space endowed with a partial ordering $\preceq$. For each $A, B \in P B(X)$, we write $A \prec_{1} B$ if for each $a \in A$, there exists $b \in P_{B}(a)$ such that $a \prec b$.

Definition 3.7 Let $(X, d)$ be a metric space endowed with a partial ordering $\preceq, g: X \rightarrow X$ be a surjective map and $T: X \rightarrow P B(X)$. Then $T$ is said to be $g$-increasing if for any $x, y \in X$,

$$
g(x) \prec g(y) \quad \Rightarrow \quad T x \prec_{1} T y .
$$

If $g$ is the identity map and $T: X \rightarrow P B(X)$ is $g$-increasing, we simply say that $T$ is increasing.

Example 3.8 Let $X=[1, \infty)$ and let $\leq$ be a usual partial ordering on $X$, that is, $x \leq y$ if and only if $y-x \geq 0$. Suppose that $d$ is a usual metric on $X$. Let $T: X \rightarrow P B(X)$ and $g: X \rightarrow X$ be defined by

$$
T x= \begin{cases}{[1,4]} & \text { if } x=1 \\ \left\{2+\frac{5}{2} x\right\} & \text { otherwise }\end{cases}
$$

and $g(x)=x^{2}$. It can be shown easily that $T$ is $g$-increasing.

Moreover, we take $g(x)=x$, then it is easy to see that $T$ is increasing.

Corollary 3.9 Let $(X, d)$ be a complete metric space endowed with a partial ordering $\preceq$, $g: X \rightarrow X$ be a surjective and $T: X \rightarrow P B(X)$ be a multi-valued mapping. Suppose that

(1) $T$ is g-increasing;

(2) there exist $x_{0} \in X$ and $u \in T x_{0}$ such that $g\left(x_{0}\right) \prec u$;

(3) for each sequence $\left\{x_{n}\right\}$ such that $g\left(x_{n}\right) \prec g\left(x_{n+1}\right)$ for all $n \in \mathbb{N}$ and $g\left(x_{n}\right)$ converges to $g(x)$, for some $x \in X$, then $g\left(x_{n}\right) \prec g(x)$ for all $n \in \mathbb{N}$;

(4) there exist $L \geq 0$ and $k, r \in[0,1)$ with $0<k+r<1$ such that

$$
H(T x, T y) \leq k d(g(x), g(y))+L D(g(y), T x)+r D(g(x), T y)
$$

for all $x, y \in X$ with $g(x) \prec g(y)$.

Then there exists $u \in X$ such that $g(u) \in T u$. 
Proof Define $G=(V(G), E(G))$, where $V(G)=X$ and $E(G)=\{(x, y): x \prec y\}$. Let $x, y \in X$ be such that $(g(x), g(y)) \in E(G)$. Then $g(x) \prec g(y)$. Since $T$ is $g$-increasing, we have $T x \prec_{1} T y$. Then, for any $u \in T x$, there exists $v \in P_{T y}(u)$ such that $u \prec v$ and so $(u, v) \in E(G)$. Thus $T$ is weak $g$-graph-preserving. By (2), there exist $x_{0} \in X$ and $u \in T x_{0}$ such that $g\left(x_{0}\right) \prec u$, and so $\left(g\left(x_{0}\right), u\right) \in E(G)$. So assumption (ii) of Theorem 3.3 is satisfied. It is easy to see that (iii) and (iv) of Theorem 3.3 are also satisfied. Therefore, this corollary is obtained directly by Theorem 3.3.

If $g$ is the identity map, then the following result is directly obtained by Corollary 3.9.

Corollary 3.10 Let $(X, d)$ be a complete metric space endowed with a partial ordering $\preceq$. Let $T: X \rightarrow P B(X)$ be a multi-valued mapping. Suppose that

(1) $T$ is increasing;

(2) there exist $x_{0} \in X$ and $u \in T x_{0}$ such that $x_{0} \prec u$;

(3) for each sequence $\left\{x_{n}\right\}$ such that $x_{n} \prec x_{n+1}$ for all $n \in \mathbb{N}$ and $x_{n}$ converges to $x$, for some $x \in X$, then $x_{n} \prec x$ for all $n \in \mathbb{N}$;

(4) there exist $L \geq 0$ and $k, r \in[0, \infty)$ with $0<k+r<1$ such that

$$
H(T x, T y) \leq k d(x, y)+L D(y, T x)+r D(x, T y)
$$

for all $x, y \in X$ with $x \prec y$.

Then there exists $u \in X$ such that $u \in T u$.

\section{Applications}

In this section, we prove the existence of a coupled fixed point for a single-valued mapping in a complete metric space endowed with a directed graph.

Let $X$ be a nonempty set and $F: X \times X \rightarrow X$ be a single-valued mapping. An element $(x, y) \in X \times X$ is called a coupled fixed point of $F$ if $x=F(x, y)$ and $y=F(y, x)$. We denote by $C \operatorname{Fix}(F)$ the set of all coupled fixed points of the mapping $F$, i.e., $C \operatorname{Fix}(F)=\{(x, y) \in$ $X \times X \mid F(x, y)=x$ and $F(y, x)=y\}$.

Coupled fixed point theorems and their application were investigated by many authors (see [12, 19, 23-27] for examples).

Definition 4.1 [12] Let $(X, \preceq)$ be a partially ordered set and $F: X \times X \rightarrow X$ be a given mapping. The mapping $F$ is said to have the mixed monotone property if it is monotone nondecreasing in $x$ and monotone nonincreasing in $y$, that is,

$$
\begin{array}{ll}
x_{1}, x_{2} \in X, \quad x_{1} \preceq x_{2} \quad \Rightarrow \quad F\left(x_{1}, y\right) \preceq F\left(x_{2}, y\right), \\
y_{1}, y_{2} \in X, \quad y_{1} \preceq y_{2} \quad \Rightarrow \quad F\left(x, y_{1}\right) \succeq F\left(x, y_{2}\right) .
\end{array}
$$

Recently, Chifu and Petrusel [28] introduced the concept of edge-preserving as follows.

Definition 4.2 [28] We say that $F: X \times X \rightarrow X$ is edge-preserving if $(x, u) \in E(G),(y, v) \in$ $E\left(G^{-1}\right)$ implies $(F(x, y), F(u, v)) \in E(G)$ and $(F(y, x), F(v, u)) \in E\left(G^{-1}\right)$. 
Let $(X, d)$ be a metric space and let $Y=X \times X$. Then the mapping $\eta: Y \times Y \rightarrow[0, \infty)$ defined by

$$
\eta((x, y),(u, v))=d(x, u)+d(y, v) \quad \text { for all }(x, y),(u, v) \in Y
$$

is a metric on $Y$, and it is easy to see that $(X, d)$ is complete if and only if $(Y, \eta)$ is complete.

For a mapping $F: X \times X \rightarrow X$, we define the mapping $T_{F}: Y \rightarrow Y$ by

$$
T_{F}(x, y)=(F(x, y), F(y, x)) \quad \text { for all }(x, y) \in Y .
$$

Note that an element $(x, y) \in X \times X$ is a coupled fixed point of $F$ if and only if $(x, y)$ is a fixed point of $T_{F}$.

Theorem 4.3 Let $(X, d)$ be a complete metric space and $G=(V(G), E(G))$ be a directed graph such that $V(G)=X$. Let the mapping $F: X \times X \rightarrow X$ be edge-preserving and suppose that the following properties hold:

(i) there exist $x_{0}, y_{0} \in X$ such that $\left(x_{0}, F\left(x_{0}, y_{0}\right)\right) \in E(G)$ and $\left(y_{0}, F\left(y_{0}, x_{0}\right)\right) \in E\left(G^{-1}\right)$;

(ii) $X$ has the following property:

(a) if any sequence $\left\{x_{n}\right\}$ in $X$ such that $x_{n} \rightarrow x$ and $\left(x_{n}, x_{n+1}\right) \in E(G)$ for $n \in \mathbb{N}$, then $\left(x_{n}, x\right) \in E(G)$ for all $n \in \mathbb{N}$;

(b) if any sequence $\left\{y_{n}\right\}$ in $X$ such that $y_{n} \rightarrow y$ and $\left(y_{n}, y_{n+1}\right) \in E\left(G^{-1}\right)$ for $n \in \mathbb{N}$, then $\left(y_{n}, y\right) \in E\left(G^{-1}\right)$ for all $n \in \mathbb{N}$;

(iii) there exist $L \geq 0$ and $k, r \in[0,1)$ with $0<k+r<1$ such that

$$
\begin{aligned}
& d(F(x, y), F(u, v))+d(F(y, x), F(v, u)) \\
& \leq k[d(x, u)+d(y, v)]+L[d(u, F(x, y))+d(v, F(y, x))] \\
& \quad+r[d(x, F(u, v))+d(y, F(v, u))]
\end{aligned}
$$

for all $x, y, u, v \in X$ with $(x, u) \in E(G)$ and $(y, v) \in E\left(G^{-1}\right)$.

Then there exist $x, y \in X$ such that $x=F(x, y)$ and $y=F(y, x)$.

Proof Let $Y=X \times X$ and $\eta$ be a metric on $Y$ given by

$$
\eta((x, y),(u, v))=d(x, u)+d(y, v) \quad \text { for all }(x, y),(u, v) \in Y .
$$

Then $(Y, \eta)$ is a complete metric space. Let $G_{Y}$ be a directed graph defined by $G_{Y}=$ $\left(V\left(G_{Y}\right), E\left(G_{Y}\right)\right)$, where $V\left(G_{Y}\right)=Y$ and

$$
E\left(G_{Y}\right)=\left\{((x, y),(u, v)) \mid(x, u) \in E(G) \text { and }(y, v) \in E\left(G^{-1}\right)\right\} .
$$

We shall show that $T_{F}$ satisfies all conditions of Theorem 3.4. Let $(x, y),(u, v) \in Y$ such that $((x, y),(u, v)) \in E\left(G_{Y}\right)$. Then $(x, u) \in E(G)$ and $(y, v) \in E\left(G^{-1}\right)$. Since $F$ is edge-preserving, we have $(F(x, y), F(u, v)) \in E(G)$ and $(F(y, x), F(v, u)) \in E\left(G^{-1}\right)$. Hence $((F(x, y), F(y, x)),(F(u, v)$, $F(v, u))) \in E\left(G_{Y}\right)$. Thus $\left(T_{F}(x, y), T_{F}(u, v)\right) \in E\left(G_{Y}\right)$. So $T_{F}$ satisfies assumption (i) of Theorem 3.4. By (2), there exists $\left(x_{0}, y_{0}\right) \in Y$ such that $\left(x_{0}, F\left(x_{0}, y_{0}\right)\right) \in E(G)$ and $\left(y_{0}, F\left(y_{0}, x_{0}\right)\right) \in$ 
$E\left(G^{-1}\right)$, so $\left(\left(x_{0}, y_{0}\right),\left(F\left(x_{0}, y_{0}\right), F\left(y_{0}, x_{0}\right)\right)\right) \in E\left(G_{Y}\right)$. Thus $\left(\left(x_{0}, y_{0}\right), T_{F}\left(x_{0}, y_{0}\right)\right) \in E\left(G_{Y}\right)$, so assumption (ii) of Theorem 3.4 is satisfied by $T_{F}$. Next, we will show that $T_{F}$ satisfies assumption (iv) of Theorem 3.4. By condition (iii), we have

$$
\begin{aligned}
\eta\left(T_{F}(x, y), T_{F}(u, v)\right)= & \eta((F(x, y), F(y, x)),(F(u, v), F(v, u))) \\
= & d(F(x, y), F(u, v))+d(F(y, x), F(v, u)) \\
\leq & k[d(x, u)+d(y, v)]+L[d(u, F(x, y))+d(v, F(y, x))] \\
& +r[d(x, F(u, v))+d(y, F(v, u))] \\
= & k \eta((x, y),(u, v))+L \eta((u, v),(F(x, y), F(y, x))) \\
& +r \eta((x, y),(F(u, v), F(v, u))) \\
= & k \eta((x, y),(u, v))+\operatorname{Ln}\left((u, v), T_{F}(x, y)\right) \\
& +r \eta\left((x, y), T_{F}(u, v)\right)
\end{aligned}
$$

for all $(x, y) \in E(G)$ and $(y, v) \in E\left(G^{-1}\right)$. So assumption (iv) of Theorem 3.4 is satisfied. Finally, we will show that $Y$ has Property (A). For any sequence $\left(x_{n}, y_{n}\right)$ in $Y$, it is easy to see that if $\left(x_{n}, y_{n}\right) \rightarrow(x, y)$ and $\left(\left(x_{n}, y_{n}\right),\left(x_{n+1}, y_{n+1}\right)\right) \in E\left(G_{Y}\right)$ for $n \in \mathbb{N}$, then $x_{n} \rightarrow x, y_{n} \rightarrow y$, $\left(x_{n}, x_{n+1}\right) \in E(G)$, and $\left(y_{n}, y_{n+1}\right) \in E\left(G^{-1}\right)$. By (ii), we have $\left(x_{n}, x\right) \in E(G)$ and $\left(y_{n}, y\right) \in E\left(G^{-1}\right)$. So $\left(\left(x_{n}, y_{n}\right),(x, y)\right) \in E\left(G_{Y}\right)$. Thus all conditions of Theorem 3.4 are satisfied. Hence there exists $(x, y) \in Y$ such that $(x, y)=T_{F}(x, y)$, which implies that $(x, y)$ is a coupled fixed point of $F$.

The following corollary is a consequence of Theorem 4.3.

Corollary 4.4 Let $(X, \preceq)$ be a partially ordered set and suppose there is a metric $d$ on $X$ such that $(X, d)$ is a complete metric space. Let $F: X \times X \rightarrow X$ be a mapping having the mixed monotone property on $X$ and there exist $x_{0}, y_{0} \in X$ such that $x_{0} \preceq F\left(x_{0}, y_{0}\right)$ and $y_{0} \succeq F\left(y_{0}, x_{0}\right)$. Assume that there exist $L \geq 0$ and $k, r \in[0,1)$ with $0<k+r<1$ such that

$$
\begin{aligned}
& d(F(x, y), F(u, v))+d(F(y, x), F(v, u)) \\
& \leq \quad k[d(x, u)+d(y, v)]+L[d(u, F(x, y))+d(v, F(y, x))] \\
& \quad+r[d(x, F(u, v))+d(y, F(v, u))]
\end{aligned}
$$

for all $x, y, u, v \in X$ with $x \preceq u$ and $y \succeq v$. Suppose that $X$ has the following property:

(a) if a nondecreasing sequence $\left\{x_{n}\right\} \rightarrow x$, then $x_{n} \preceq x$ for all $n \in \mathbb{N}$;

(b) if a nonincreasing sequence $\left\{y_{n}\right\} \rightarrow y$, then $y_{n} \succeq y$ for all $n \in \mathbb{N}$.

Then there exist $x, y \in X$ such that $x=F(x, y)$ and $y=F(y, x)$, that is, $F$ has a coupled fixed point in $X \times X$.

Proof Let $G=(V(G), E(G))$, where $V(G)=X$ and $E(G)=\{(x, y) \mid x \preceq y\}$. We can directly check that all conditions of Theorem 4.3 are satisfied. Therefore $F$ has a coupled fixed point. 
Authors' contributions

All authors contributed equally and significantly in writing this article. All authors read and approved the final manuscript.

\section{Acknowledgements}

The authors would like to thank the Thailand Research Fund under the project RTA5780007 and Chiang Mai University, Chiang Mai, Thailand for the financial support.

Received: 10 February 2015 Accepted: 10 July 2015 Published online: 28 July 2015

References

1. Banach, S: Sur les opérations dans les ensembles abstraits et leur application aux équations itégrales. Fundam. Math. 3, 133-181 (1922)

2. Berinde, V: Approximating fixed points of weak contractions using the Picard iteration. Nonlinear Anal. Forum 9(1), 43-53 (2004)

3. Bojor, F: Fixed point theorems for Reich type contraction on metric spaces with a graph. Nonlinear Anal. 75 3895-3901 (2012)

4. Branciari, A: A fixed point theorem for mappings satisfying a general contractive condition of integral type. J. Math. Anal. Appl. 29, 531-536 (2002)

5. Chatterjea, SK: Fixed point theorems. C. R. Acad. Bulgare Sci. 25, 727-730 (1972)

6. Kannan, R: Some results on fixed points. Bull. Calcutta Math. Soc. 10, 71-76 (1968)

7. Nieto, JJ, Rodriguez-Lopez, R: Contractive mapping theorems in partially ordered sets and applications to ordinary differential equation. Order 22, 223-239 (2005)

8. Nieto, JJ, Rodriguez-Lopez, R: Existence and uniqueness of fixed point in partially ordered sets and applications to ordinary differential equation. Acta Math. Sin. Engl. Ser. 23(12), 2205-2212 (2007)

9. Ran, ACM, Reurings, MCB: A fixed point theorem in partially ordered sets and some applications to matrix equations. Proc. Am. Math. Soc. 132, 1435-1443 (2004)

10. Zamfirescu, T: Fixed point theorems in metric spaces. Arch. Math. (Basel) 23, 292-298 (1972)

11. Agarwal, RP, El-Gebeily, MA, O’Regan, D: Generalized contractions in partially ordered metric spaces. Appl. Anal. 87, 1-8 (2008)

12. Gnana Bhaskar, T, Lakshmikantham, V: Fixed point theorems in partially ordered metric spaces and applications. Nonlinear Anal. TMA 65, 1379-1393 (2006)

13. Nadler, S: Multi-valued contraction mappings. Pac. J. Math. 20(2), 475-488 (1969)

14. Berinde, M, Berinde, V: On a general class multi-valued weakly Picard mappings. J. Math. Anal. Appl. 326, 772-782 (2007)

15. Jachymski, J: The contraction principle for mappings on a metric with a graph. Proc. Am. Math. Soc. 139, 1359-1373 (2008)

16. Beg, I, Butt, AR: Fixed point for set valued mappings satisfying an implicit relation in partially ordered metric spaces. Nonlinear Anal. 71, 3699-3704 (2009)

17. Alfuraidan, MR: Fixed points of multivalued mappings in modular function spaces with a graph. Fixed Point Theory Appl. 2015, Article ID 42 (2015). doi:10.1186/s13663-015-0292-7

18. Alfuraidan, MR: Remarks on monotone multivalued mappings on a metric space with a graph. J. Inequal. Appl. (2015). doi:10.1186/s13660-015-0712-6

19. Nicolae, A, O'Regan, D, Petrusel, A: Fixed point theorems for single-valued and multi-valued generalized contractions in metric spaces endowed with a graph. Georgian Math. J. 18, 307-327 (2011)

20. Dinevari, T, Frigon, T: Fixed point results for multi-valued contractions on a metric space with a graph. J. Math. Anal. Appl. 405, 507-517 (2013)

21. Tiammee, J, Suantai, S: Coincidence point theorems for graph-preserving multi-valued mappings. Fixed Point Theory Appl. (2014). doi:10.1186/1687-1812-2014-70

22. Phon-on, A, Sama-Ae, A, Makaje, N, Riyapan, P, Busaman, S: Coincidence point theorems for weak graph preserving multi-valued mapping. Fixed Point Theory Appl. (2014). doi:10.1186/1687-1812-2014-248

23. Agarwal, RP, Karapınar, E: Remarks on some coupled fixed point theorems in G-metric spaces. Fixed Point Theory Appl. 2013, Article ID 2 (2013)

24. Mursaleen, M, Mohiuddine, SA, Agarwal, RP: Coupled fixed point theorems for $\alpha$ - $\psi$-contractive type mappings in partially ordered metric spaces. Fixed Point Theory Appl. 2012, Article ID 228 (2012)

25. Roldán, A, Martínez-Moreno, J, Roldán, C, Karapınar, E: Some remarks on multidimensional fixed point theorems. Fixed Point Theory 15(2), 545-558 (2014)

26. Urs, C: Coupled fixed point theorems and applications to periodic boundary value problems. Miskolc Math. Notes 14(1), 323-333 (2013)

27. Urs, C: Ulam-Hyers stability for coupled fixed points of contractive type operators. J. Nonlinear Sci. Appl. 6, 124-136 (2013)

28. Chifu, C, Petrusel, G: New results on coupled fixed point theory in metric spaces endowed with a directed graph. Fixed Point Theory Appl. 2014, Article ID 151 (2014) 